\title{
Photoinduced Conversion of Hybridization in Graphite
}

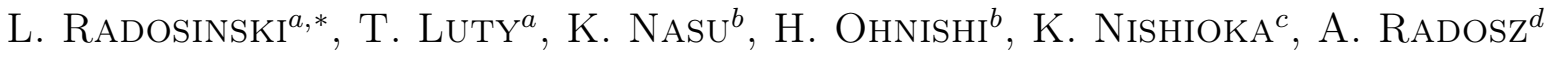 \\ AND D. WÓJT ${ }^{d}$ \\ ${ }^{a}$ Institute of Theoretical and Chemical Physics, Wrocław University of Technology \\ Wybrzeże Wyspiańskiego 27, 50-370 Wrocław, Poland \\ ${ }^{b}$ High Energy Accelerator Research Organization (KEK), 1-1 Oho, 030-0805 Tsukuba, Japan \\ ${ }^{c}$ Department of Theoretical and Computational Molecular Science, Institute for Molecular Science \\ Okazaki Aichi, 444-8585, Japan \\ ${ }^{d}$ Institute of Physics, Wrocław University of Technology, Wybrzeże Wyspiańskiego 27, 50-370 Wrocław, Poland

\begin{abstract}
Recent experiments indicate that a photostimulated graphite with a femtosecond laser pulse results in the formation of a stable domain with $s p^{3}$ like interlayer bonds. By means of the energy barrier minimization and molecular dynamics using the empirical Brenner potential we study a geometrical structure of the new phase. We clarify proliferation of the initial domain and prove that the overall process is a multiphoton one. Furthermore, we present a model describing the initial transformation - an interlayer charge transfer resulting in the localization of an exciton-like state. The local density approximation electronic structure analysis reveals that the electronic state of the new phase is an insulator immersed in semimetal. We study by means of the long-range carbon bond order potential the effect of the existence of the new phase on the surrounding graphite and propose a new mid step structure on the path of a photoinduced graphite-diamond conversion.
\end{abstract}

PACS: 81.30.Hd, 61.66.-f

\section{Introduction}

Rapidly growing interest in carbon based materials is associated with its particular properties. Carbon develops variety of phases with $s p, s p^{2}$ and $s p^{3}$ hybridizations. Interesting aspect of different hybridizations in carbon based materials is a relation between a graphite, with a dominating $s p^{2}$ hybridization, and a diamond characterized by $s p^{3}$ hybridization $[1,2]$. The energy barrier between the graphite and the diamond turns out to be $0.3 \mathrm{eV} /$ atom $[1,2]$. This rather large value is mainly due to the $s p^{2} \rightarrow s p^{3}$ inter-layer bond conversion. The inter-layer distance in the graphite is large $(3.35 \AA)$ compared to the distance of intra-layer bonds $(1.42 \AA)$. Hence in the case of the direct transition in order to create $\sigma$ -type bonds between the layers, a large amount of energy is required. This fact is manifested in experimental approaches wherein mostly high pressure and high temperature $\left(3000^{\circ} \mathrm{C}, 15 \mathrm{kPa}\right)[3,4]$ or strong X-ray beams [5] are used for the direct transformation. In such a direct synthesis, a large amount of energy is distributed over the crystal, resulting in a global, simultaneous conversion of a macroscopic number of carbon atoms.

Recent experiments [6-8] indicated another perspective of a graphite-diamond relationship. The existence

* corresponding author; e-mail: lukasz.radosinski@pwr.wroc.pl of a metastable phase(s) characterized by local $s p^{3}$-type hybridization was revealed. Two teams (see $[6,7]$ and [8]) reported a photogeneration of a non-equilibrium long-lived phases with inter-layer $\sigma$ bonds between two distorted graphite layers. The photogenerated phase, of a nanoscopic size, carrying the imprints of graphite and diamond phases, is called a diaphite. In the first experiment $[6,7]$, illumination by femtosecond pulse of laser light with the energy $1.57 \mathrm{eV}$ polarized perpendicularly to the graphite led to a new buckling domain. In this case a carbon ring was deformed in such a way that 4 atoms were extruded out of the layer and 2 were intruded inside of the layered crystal - this phase was stable for several days at room temperature. In the other experiment [8] another structural change in the graphite was induced by a femtosecond laser pulse irradiation. Following an initial contraction of the interlayer spacing by less than $6 \%$, the graphite was driven nonthermally into a transient new state involving interlayer bonds. This led to the contraction of the interlayer distance from the initial $3.35 \AA$ (graphite) to the final $1.9 \AA$ one in a new, photogenerated phase.

Such transformations appear to belong to the class of the so-called photoinduced phase transition (PIPT) [9-12]. If it is so, we are uncovering a new aspect of PIPT. Usually, a photoexcited electron in an insulating crystal induces a local lattice distortion and such a sudden change of the charge distribution ultimately results 
in new equilibrium atomic positions within the excited state. This idea is associated with the hidden multistability: besides its true ground state, a material also possesses a false, metastable one [9]. One can assume that ground and metastable states are separated by a high energy barrier, being inaccessible by thermal fluctuations; due to photoexcitation and lattice relaxation, the system may jump from the ground state to the metastable one, resulting in a new electronic and structural order. This type of a non-equilibrium phase transition has been observed recently in many organic molecular solids, organic metal-complexes crystals and in the Perovskite type compounds [9]. Nonetheless, the photogenerated phase transformation observed in graphite [6-8] differs from conventional PIPT. Key issue is the fact that graphite is not an insulating material and the PIPT scenario as described above does not apply straightforwardly.

The aim of this paper is to present in detail the methods and the results of theoretical investigations of different possibilities of generation of novel domains, the diaphite, consisting of locally dimerized graphite layers with $\sigma$-type inter-layer bonds. We will present results of examination of several possible candidates for such domain type structures. The one corresponding to the most stable configuration will be indicated. The transformation path following evolution of the initial, graphite phase, step by step along the potential energy surface, will be described. A photogenerated graphite-diaphite conversion will be considered in the context of photoinduced phase transformations: dynamics of this phase conversion will be analyzed within a simplified scenario of the processes at early stages. In this scenario photoexcitation leads to a charge transfer from a one graphite layer to its neighboring one, resulting in the electron-hole pair creation. This exciton-like pair should be dissipated but as it is shown here, there is a small, but finite probability that the excitation will remain, at the same site. The ultimate result is graphite dimerization.

The structure of the paper is as follows: in the first section Brenner's potential calculations revealing geometrical parameters of the minimal diaphite domain are presented, in the second section local density approximation (LDA) calculations revealing the electronic structure of the diaphite are described, in the third section a model describing initial transformation is formulated followed by molecular dynamics calculations for the diaphite domain proliferation. The paper ends with long-range carbon bond order potential (LCBOP) calculations for a new mid step material on the path of graphite-diamond phototransformation.

To study adiabatic the potential energy surface one can begin with two, initially non-interacting graphite layers with the ABAB-type stacking. On the experimental ground it is supposed that a new domain, is immersed in the semi-metallic graphite and the number of atoms involved in this transformation will be about 1000. As the total number of the surrounding original graphite has to be of an order of magnitude higher, it should comprise
$10^{4}$ atoms. To handle such a great number of atoms without translational symmetry, methods different than the LDA should be applied. We have been using modified Brenner's approach [13]. This semi-empirical potential has been deviced for and is widely used in various problems involving carbon and hydrocarbon based clusters. In contrast to the Lennard-Jones two-body type potential theory, the Brenner potential takes 3- and 4-body effects into account as a change of an angle between each bond or as a change of the coordination number of the each specific atom. The values of parameters of this potential are determined so that they take into account almost all existing experimental and theoretical data of carbon clusters [13].

We would like to emphasize that the graphite-diaphite phase conversion is different from (global) phase transitions, as it is a local and a non-equilibrium one. The main part of the crystal still remains in its original semimetallic phase, and a newly photogenerated domain with the interlayer $\sigma$-bonds is immersed in it, breaking the translational symmetry of the crystal lattice. The energy barrier to create this new domain is strongly affected by the domain boundary which causes a significant energy increase. On the contrary, in the case of the conventional global and simultaneous transformations, one can assume that some translational symmetry of the crystal lattice still remains preserved even at the top state of the energy barrier of the phase transition.

\section{The minimal structure}

\subsection{Brenner's potential}

In order to clarify what is the geometrical structure of the diaphite we have performed total cohesive-energy calculations followed by a graphite-diaphite barrier height minimization. Since the size of the diaphite domain is of the order of $10^{5}$ carbon atoms and does not have translational symmetry, $a b$ initio methods like the LDA are ineffective. Hence we use 3-, 4-body empirical bond order Brenner's potential [13].

Brenner's method in calculations of the cohesive energy of the carbon based-material is defined as:

$$
E=\sum_{i} E_{i}
$$

where $E_{i}$ is a contribution from the atom $i$, is based on the assumption about the distinction of short- and long range potential for the each carbon atom

$$
E_{i}=\sum_{j(<i)}\left[V_{\mathrm{R}}\left(r_{i j}\right)-\bar{B}_{i j} V_{\mathrm{A}}\left(r_{i j}\right)\right] .
$$

The sum (2) is taken over the bonds of length $r_{i j}$ between $j$-th and $i$-th, atoms and it consists of the attractive $V_{\mathrm{A}}\left(r_{i j}\right)$ and the repulsive $V_{\mathrm{R}}\left(r_{i j}\right)$, coupling within the bond, respectively.

Although Brenner's potential does not take into account long range $(>2 \AA)$ interactions it does not cause serious mismatch. The transformation occurs only in limited volume (2 layers, 200 atoms each) and the energy 
difference due to long range interactions is minor. In such case the energy contribution due to long range interactions is several orders smaller than due to inter-layer bond formation. Hence in our calculations we focus our attention to a predominant process governing the transformation and use of the Brenner potential seems appropriate.

\subsection{The intruded domain}

Initially non-interacting, two graphite layers $(200 \times 200$ neutral carbon atoms), whose ends are connected by the periodic boundary condition, undergone deformation. In order to study the energy landscape we consider a class of deformation patterns imposed on the system. Namely, an interatomic distance $r_{i j}$ :

$$
r_{i j} \equiv\left|\boldsymbol{r}_{i}-\boldsymbol{r}_{j}\right|
$$

is expressed in terms of $\boldsymbol{r}_{i}$ describing a shift of the $i$-th atom from its original position $\left(\equiv \boldsymbol{r}_{i 0}\right)$ in the graphite as

$$
\boldsymbol{r}_{i} \equiv \boldsymbol{r}_{i 0}+E_{n}\left(\boldsymbol{r}_{i 0}\right)\left[\left(\Delta q+\delta B_{d}\left(\boldsymbol{r}_{i 0}\right)\right) \overline{\boldsymbol{e}}_{z}+\Delta X \overline{\boldsymbol{e}}_{x}\right]
$$

One assumes that the deformation $E_{n}\left(\boldsymbol{r}_{i 0}\right)$ is of an envelope shape, given as

$$
E_{n}\left(\boldsymbol{r}_{i 0}\right)=0.5\left[\tanh \left(\theta|| \boldsymbol{r}_{i 0}-\boldsymbol{e}_{\boldsymbol{z}}\left(\boldsymbol{e}_{\boldsymbol{z}} \cdot \boldsymbol{r}_{i 0}\right)\left|-L_{0}\right|\right)-1\right] .
$$

Here $\boldsymbol{e}_{x}, \boldsymbol{e}_{z}$ are the unit vectors in the $x$ and $z$ axes, respectively. Parameters given in Eq. (4) are as follows (see Fig. 1): $\Delta q$ - the amplitude of the planar deflation, $L_{0}$ - the size of the intruded domain, $\theta-$ the domain boundary width and $\Delta X$ - the magnitude of the shear displacement; $B_{d}\left(\boldsymbol{r}_{i 0}\right)$ in Eq. (4) denotes the buckling pattern within the six-membered ring, and it will be defined further in the text. In Fig. 2, this buckling is symbolically denoted by a zigzag line.

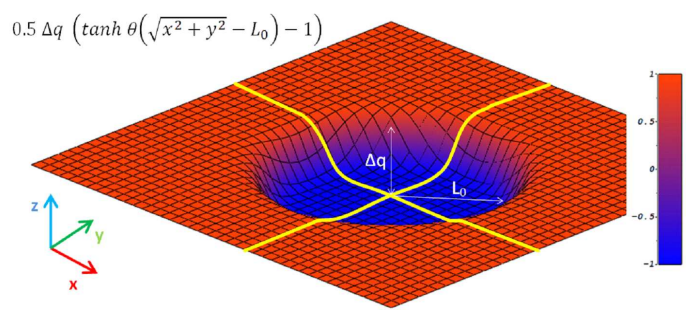

Fig. 1. The distortion pattern used in the calculations: $\Delta q-$ the planar intrusion amplitude, $\delta$ - the buckling amplitude, $L_{0}$ - the system size, $\theta$ - the shape coefficient.

The intruded domain is characterized by a buckling perpendicular to the layer of an amplitude $\delta$; it will also be assumed that the center of mass of the six-membered ring is preserved. The deformation of the other layer is symmetric as shown in Fig. 2. Using Brenner's method, one can calculate the adiabatic energy of the domain formation. The key features of our calculations are: the height of the barrier, the point of the top of the barrier and the depth of the potential well corresponding to the

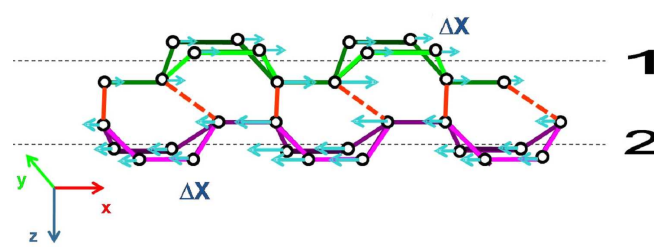

Fig. 2. The modulation pattern applied in calculations with the additional shear displacement; two atoms forming regular line along the $x$ axis direction are intruded with an amplitude $\delta$, remaining atoms are extruded with the amplitude $0.5 \delta$. In order to optimize the inter-layer bond angles, both deflated domains are shifted relatively in the $x$ direction, as denoted $\Delta X(>0)$.

metastable phase. The stable point formation is a result of competition of two processes: the $\sigma$-type inter-layer bond formation resulting in an energy gain, and the $s p^{2}$ type intra-layer bond tension resulting in an energy loss. As mentioned before, the energy required for the direct $s p^{2} \rightarrow s p^{3}$ transition is rather high. The reason is a long distance between neighboring layers (3.35 $\AA$ ), while the inter-layer bond formation starts only at a short distance $(\approx 2 \AA[14])$. Hence the displacement of the carbon atoms necessary to create inter-layer $\sigma$-type bonds takes considerable amounts of energy, if it occurs simultanously all over the crystal. In this case the distance is reduced within the locally intruded domain, so that, the inter-layer bonds are created quite easily resulting in a lower energy barrier. The energetic cost of shortening the distance between the layers is spread over as a gradual tension at the border of the domain, and becomes much lower than in the case of a direct and global motion.

Using the aforementioned pattern defined by Eqs. (4), (5) and the Brenner potential, we have performed the total cohesive energy calculations. By varying values of selected parameters $\left(\Delta q, \delta, \theta, L_{0}\right)$ we have obtained a potential energy surface (PES) of the conversion. The extremal points indicate stable configurations of distorted structures. Our goal is to find values of parameters $\left(\Delta q, \delta, \theta, L_{0}\right)$ minimizing the energy barrier between the initial graphite structure and a new one corresponding to a new stable point.

The adiabatic energy calculations [15] revealed that a small diaphite domain appears at $1.7 \mathrm{eV}$ and the energy barrier between graphite and the new phase is about $2 \mathrm{eV}$. Once the energy barrier is overtaken a number of new stable structures are possible. However, we may divide them into two groups: $S$ type with a small intrusion and a large modulation and $L$ type with a large intrusion and a small modulation. Both types of the structure are characterized with a relatively large shear displacement $0.2 \AA$ as presented in Fig. 3 .

A third type of the diaphite structure $(L L)$ is presented in Fig. 4. This type of structure combines features of the $S$ and the $L$ type i.e. it has a large intrusion, a large modulation and a large shear displacement introducing a new symmetry to the system. One may find that this 


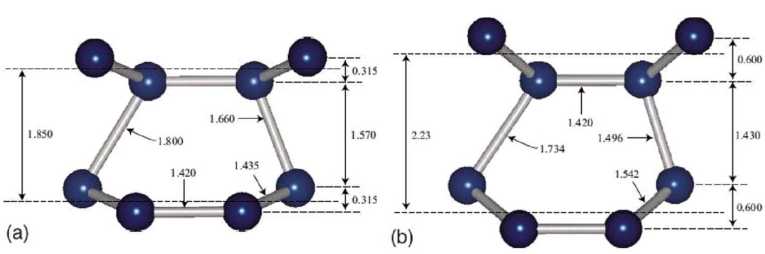

Fig. 3. Two types of the diaphite structure [14]: (a) the $S$ type structure, (b) the $L$ type structure.

type of structure is closely geometrically related with a hexagonal diamond.

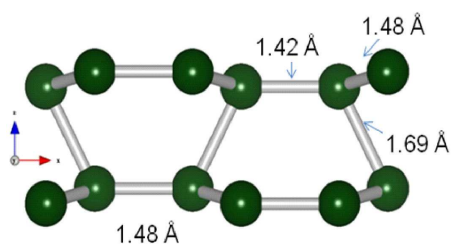

Fig. 4. Third type $(L L)$ of the diaphite structure with large modulation, intrusion and shear displacement.

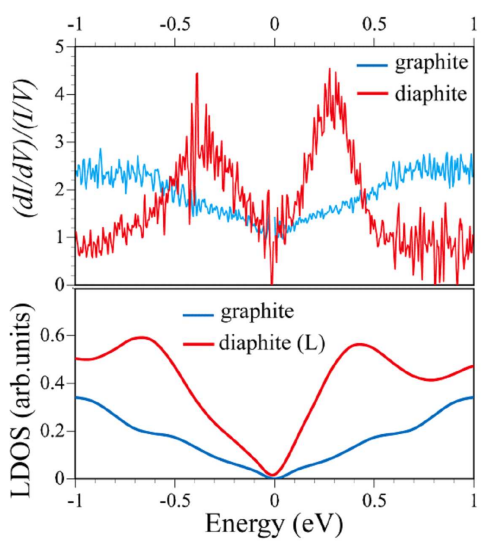

Fig. 5. Local density of the state of the diaphite domain [15].

The electronic structure of the diaphite domain is estimated, by means of the tight-binding model, in that parameters are estimated from the $a b$ initio LDA calculation, and the environment dependence is taken into account by the Hurrison law [16]. Details of the method are given in [15]. As seen in Fig. 3, two kinds of carbons exist in the diaphite structure. One is the carbons that are shifted up from the graphite surface and construct the cis-type chain at the diaphite surface. The other is carbons that are sunk down from the graphite surface and construct the $s p^{3}$-bond with the adjacent layer. As discussed in [15], the former carbons is still $s p^{2}$-like and weak $\pi$-bonds are formed. As the result, the electronic state is still semimetallic. On the other hand, latter carbons form the interlayer bond and their electronic state is $s p^{3}$-like. Hence, the diaphite domain has the $s p^{2}-s p^{3}$ mixed electronic state, since above two kinds of carbons are correlated with each other. Furthermore, the diaphite domain is always immersed in the vast graphite. Hence, the electronic state of the diaphite domain is characterized as the insulator immersed in the semimetallic continuum of the graphite. The electronic state of the surface carbon of the diaphite domain is compared with the experimental one, that is obtained from the scanning tunneling microscope (STM) measurement. As seen in Fig. 5, our theoretical density of states (DOS) with the aforementioned $L$-type structure well reproduces whole tendency of the experimental one. Hence, the experimentally observed diaphite domain will possess the $L$-type structure.

\section{The photoexcitation and its early dynamics}

The adiabatic energy landscape in the former section was established by considering the deformed phase and looking for its local minima corresponding to the metastable states. This is a phenomenological approach based on the experimental observation of the photoexcited, metastable, phase(s). This is a usual argument applied in the context of the photoinduced phase transformations. In this case, contrary to the usual conditions of the strongly anisotropic, isolating systems revealing photoinduced phase transitions, graphite is a conductor (and also anisotropic but two-dimensional, not one-dimensional system). Therefore the question of proliferation of the photoexcitation appears to be quite nontrivial and below we will discuss it limiting ourselves to the early stage dynamics.

\subsection{Model Hamiltonian}

We shall consider the photoexcitation in the two, initially undistorted graphite layers. During photoexcitation an electron is transferred between the layers and as a result the hole is created. In this way an exciton-like state is formed across the layers. As the graphite is a conductor, the electron and the hole would soon disappear as free carriers. However, owing to a Coulomb interaction and an electron-phonon interaction, the layers would contract, enabling $\sigma$-type-bond formation. Such a scenario would occur if the electron-hole pair remains at the same site.

Let us estimate if the probability of such a process would be nonzero. We will start from a simplified model Hamiltonian of the interacting electron-hole pair, coupled to the two-layer lattice with $N(\gg 1)$ sites in each layer

$$
H=H_{\mathrm{eh}}+H_{\mathrm{ph}}+H_{\mathrm{c}}, \hbar=1 .
$$

The first term of this Hamiltonian is the energy band of the electron or the hole, given as

$$
H_{\mathrm{eh}}=\sum_{i=1,2} \sum_{j=1,2} \sum_{k} E(k) a_{k i j}^{+} a_{k i j},
$$

where $a_{k i j}^{+}$is a usual electron, $i=1$ (hole, $i=2$ ) creation operator, the $j$ index $(=1,2)$ stands for a layer 
number, and the $k$ index denotes the two-dimensional wave vector $k=\left(k_{x}, k_{y}\right)$. We are interested in the visible excitation energy range around the Fermi level $( \pm 4 \mathrm{eV})$, and we have assumed a V-shaped electron band and the hole one centered at the $\Gamma$ point $(k=0)$ for simplicity $E(k)$ :

$$
E(k)=\sqrt{k_{x}^{2}+k_{y}^{2}}, \quad\left|k_{x}\right| \leq \pi, \quad\left|k_{y}\right| \leq \pi,
$$

where spin is neglected. In the graphite crystal, the Fermi level is at the $K$ point but this difference has no effect for our final conclusion. $H_{\mathrm{ph}}$ denotes a dispersionless, Einstein phonon localized on a lattice site $l$ with an energy $\omega$ and is given as

$$
H_{\mathrm{ph}}=\omega \sum_{l}\left(\frac{1}{2}+b_{l}^{+} b_{l}\right),
$$

where $b_{l}^{+}$denotes the creation phonon operator. The value of the energy of the optical excitation $\omega$ may be self-consistently estimated within our approach. Taking the value of the energy barrier height as $1 \mathrm{eV}$, corresponding to $0.8 \AA$ shift, one finds a restoring force coefficient,

$$
k \propto \frac{2}{0.64}\left[\frac{\mathrm{eV}}{\AA^{2}}\right] .
$$

This value corresponds to the energy of an optical mode of the carbon atom,

$$
\bar{\omega}^{2} \propto 0.4 \times 10^{28}\left[1 / \mathrm{s}^{2}\right] .
$$

So the corresponding energy

$$
\begin{aligned}
\hbar \bar{\omega} & \propto 1.1 \times 10^{-34} \mathrm{~J} \mathrm{~s} \times 0.6 \times 10^{14}\left[\frac{1}{\mathrm{~s}}\right] \\
& \propto 0.07 \times 10^{-19}[\mathrm{~J}] \propto 0.05[\mathrm{eV}]
\end{aligned}
$$

is not much different from the one experimentally observed, i.e. $0.025 \mathrm{eV}$. In our calculations we are taking the value of this parameter, $0.1 \mathrm{eV}$, according to the level of magnitude.

$H_{\mathrm{c}}$ is the electron-hole Coulomb interaction Hamiltonian given as

$$
H_{\mathrm{c}}=-\sum_{l} U\left(Q_{l}\right) n_{l 11} n_{l 22}
$$

where $Q_{l}$ is the phonon dimensionless coordinate and is defined as:

$$
Q_{l} \equiv \frac{b_{l}^{+}+b_{l}}{\sqrt{2}} .
$$

Since only this $Q_{l}$ describes the inter-layer contractions starting from the original graphite, we have only a one mode per lattice site $l$, although there are two square lattices for the electron and the hole. In the above equation, $n_{l i}=a_{l i}^{+} a_{l i}$ denotes the number operator on site $l$ and layer $i$ and

$$
a_{l i j}=\frac{1}{\sqrt{N}} \sum_{k} \mathrm{e}^{\mathrm{i} \boldsymbol{k} \cdot \boldsymbol{l}} a_{k i j} .
$$

$U\left(Q_{l}\right)$ is the electron-hole coupling strength. We assume a linear relation

$$
U\left(Q_{l}\right)=U_{0}+U_{d} Q_{l} .
$$

Its initial value is $U_{0}=1.5 \mathrm{eV}$. It corresponds to the electron-hole coupling in the undistorted graphite system. The Coulomb attraction energy between the electron and the hole was calculated, by using the MatagaNishimoto formula [17]. The real value of contraction may be derived by relating phonon energy with the energy of the top of the barrier. At the top, $Q_{l}$ takes $\bar{Q}_{l}$ value and is given as

$$
\frac{\omega}{2} \bar{Q}_{l}^{2}=0.8[\mathrm{eV}]
$$

and gives $\bar{Q}_{l}=4$. On the other hand, again applying the Mataga-Nishimoto formula [16] the electronhole Coulomb coupling strength at this point becomes $\bar{U}\left(\bar{Q}_{l}\right)=2.4 \mathrm{eV}$. Hence $U_{d}$ is determined, $U_{d}=0.225 \mathrm{eV}$. It should be noted that the present calculations were also performed with various values of $U_{0}$ ranging from $1.0 \mathrm{eV}$ to $1.6 \mathrm{eV}$, having no influence on the final conclusions.

\subsection{Calculations}

We define a Bloch state composed of an electron at the site $l+\Delta$ of the first layer, a hole of the site $l$ of the second layer, and $m$ phonons at the site $l$, as

$$
\begin{aligned}
|\Delta, m\rangle & =\frac{1}{\sqrt{N}} \sum_{l} \mathrm{e}^{-\mathrm{i} \boldsymbol{k}_{\mathrm{ph}} \cdot \boldsymbol{l}} a_{l+\Delta, 11}^{+} a_{l, 22}^{+} \frac{\left(b_{l}^{+}\right)^{m}}{\sqrt{m !}}|0\rangle, \\
|0\rangle \rightarrow \text { vacuum. } &
\end{aligned}
$$

The expression inside the sum over $l$, is an electronhole phonon state localized at the site $l$, wherein $\boldsymbol{k}_{\mathrm{ph}}$ is the wave vector of photon that creates the excitation, and we have also assumed that the phonon number $m$ is zero $(m=0)$, if the electron and the hole spanning two layers are not in the same site $(\Delta \neq 0)$. The wave vector $\boldsymbol{k}_{\mathrm{ph}}$ of a visible photon is almost zero compared to the wave vectors of the electron, the hole, or phonons in the first Brillouin zone. Henceforth, the electron-holephonon coupled states which are created by a visible photon have the full translation symmetry

$$
|\Delta, m\rangle=\frac{1}{\sqrt{N}} \sum_{l} a_{l+\Delta, 11}^{+} a_{l, 22}^{+} \frac{\left(b_{l}^{+}\right)^{m}}{\sqrt{m !}}|0\rangle .
$$

The expression inside the sum over $l$ is an electron-hole phonon state localized at around the site $l$. We can also see that in Eq. (16), $\Delta$ stands for the electron-hole relative distance. This is just the inter-layer charge transfer and the self-localized exciton.

Using this type of state as a base we can formulate eigenvalue problem of $H$. To do this we calculate matrix elements of the $\left\langle m, \Delta|H| \Delta^{\prime}, m^{\prime}\right\rangle$ followed by its diagonalization.

For practical purpose we calculate transfer energies in real space rather than the wave vector one

$$
T(l)=\frac{1}{N} \sum_{k} E(k) \mathrm{e}^{\mathrm{i} \boldsymbol{k} \cdot \boldsymbol{l}} .
$$

Since $T(l)$, thus defined, has a long tail in the real space, 
we decided to truncate it at $l=10$. Finally, we apply a torus type periodic boundary conditions for relative electron-hole distance. Using the state defined by the formula (16) as basis, matrix elements $\left\langle m, \Delta|H| \Delta^{\prime}, m^{\prime}\right\rangle$ of the Hamiltonian $H$ have been calculated.

Diagonalizing matrix $\left\langle m, \Delta|H| \Delta^{\prime}, m^{\prime}\right\rangle$ we obtain a set of eigenvalues and eigenfunctions. In our calculations, we have chosen net $50 \times 50$ sites with 60 phonons. Having a set of eigenstates $|r\rangle$ and corresponding eigenvalues $E_{r}$, one can now calculate the temporal evolution of the coupled electron-hole phonon state using the following relation:

$$
|W(t)\rangle=\sum_{r} \mathrm{e}^{-\mathrm{i} t E_{r}}|r\rangle\langle r \mid \Delta, 0\rangle .
$$

Using $|\Delta, 0\rangle$ as the initial state we may investigate states with various initial electron-hole separations $\Delta$ while the initial phonon number is zero; $m=0$. Our main interest is to calculate probability that the electron-hole will remain at the same site $\Delta=0$. This probability is given by $D(t)$, defined as:

$$
D(t)=\left\langle W(t)\left|\left[\sum_{m}|0, m\rangle\langle 0, m|\right]\right| W(t)\right\rangle .
$$

We also compute the average phonons number as

$$
M(t)=\left\langle W(t)\left|\left[\sum_{m}|0, m\rangle m\langle 0, m|\right]\right| W(t)\right\rangle / D(t) .
$$

Calculations results for the initial quarter period are presented in Figs. 6 and 7.

In Fig. 6, the time dependence of exciton probability that it will still remain in the same site is shown. One can notice that even if $\Delta=0$, it decreases rapidly from value 1 , which reflects the free carrier relaxation process characteristic of a good conductor. However it does not reach zero but stabilizes at around 0.1 , which is nothing else but a self-localization of the electron and the hole pair. At the same time, the number of phonons rapidly increases. It means that the inter-layer contraction has been generated together with the self-localization. We have also calculated a largely distant electron-hole evolution by taking as the initial state $|\Delta, 0\rangle$ for $\Delta=10$ and 20 . We can see that these states can never localize.

\section{The diaphite proliferation and a shear displacement saturation}

After the self-localization of the electron-hole pair, the subsequent interlayer bond formation can be taken into account in a classical picture due to the translational symmetry breaking of the excited state [14]. The two carbons spanning two layers at the self-localized point are intruded inside between the two layers. These two carbons have a kinetic energy and a potential energy (a velocity and displacement toward the inside). In this stage, we approximately replace the whole state by the classical motion of carbons. The initial condition is the inward displacement and velocity of the two carbons. The original energy donated at the Franck-Condon excitation is

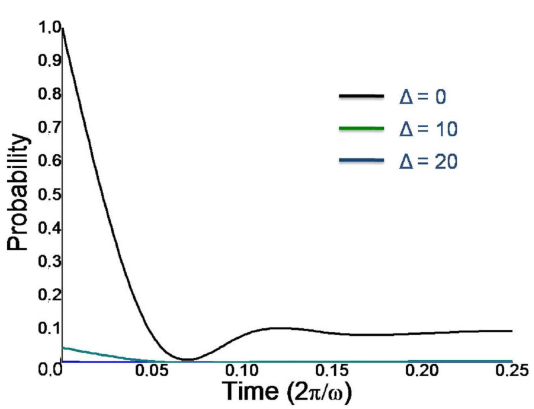

Fig. 6. The probability that the exciton remains at the same site with respect to time (in $2 \pi / \omega$ units). Different colors indicate different relative electron-hole distances: black $\Delta=0$ (top curve), green $\Delta=10$ (middle curve), blue $\Delta=20$ (bottom curve) [21].

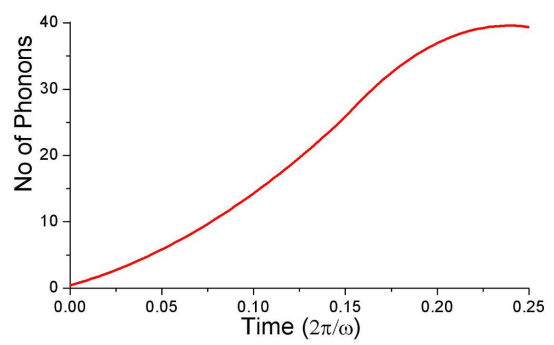

Fig. 7. The average number of phonons created during the process of the electron-hole localization (in $2 \pi / \omega$ units) [21].

converted into the distortion and the velocity of carbon atoms. Because of the quantum uncertainty, these initial conditions should reflect a large uncertainty $\pm 1.8 \mathrm{eV}$ around the given Frank-Condon energy $3.3 \mathrm{eV}$. Namely the energy of the initial state $|0,0\rangle$ is given by

$$
E_{0,0} \equiv\langle 0,0|H| 0,0\rangle=\sum_{n}|\langle 0,0 \mid n\rangle|^{2} E_{n} .
$$

One may calculate the mean-energy width as

$$
\Delta E_{0,0} \equiv \sqrt{\sum_{n}\left[|\langle 0,0 \mid n\rangle|\left(E_{n}-E_{0,0}\right)\right]^{2}}
$$

resulting $\pm 1.8 \mathrm{eV}$. In this section, by using a classical molecular dynamics, we review how the inter-layer bonds are formed $[14,18]$. Here, we also adopt the semiempirical Brenner potential in the molecular dynamics (MD) calculations. The system consists of two graphite layers (AB stacking) with the initial interlayer distance $3.35 \AA$, and each layer includes 6240 carbons in about $128 \AA \times$ $128 \AA$, wherein a periodic boundary condition is imposed.

First, we see the formation of one inter-layer bond at a self-localized site. We assume that the initial displacements and initial velocities of the two carbons are in opposite direction, but have the same magnitude. This initial kinetic energy is given by $K_{0}=2\left(m_{\mathrm{c}} v_{0}^{2} / 2\right)$, where $m_{\mathrm{c}}$ is the mass of a carbon atom and $v_{0}$ is the initial velocity. The initial potential energy is obtained from the 
relation between the intrusion and the potential energy calculated by using the Brenner theory. Typical results of this MD calculation are shown in Fig. 8. The case of $K_{0}=3.2(2.4) \mathrm{eV}$ corresponds to an example that an interlayer bond is (not) formed successfully, where the initial potential energy is $1.54 \mathrm{eV}$ (the intrusion of $0.4 \AA$ ).
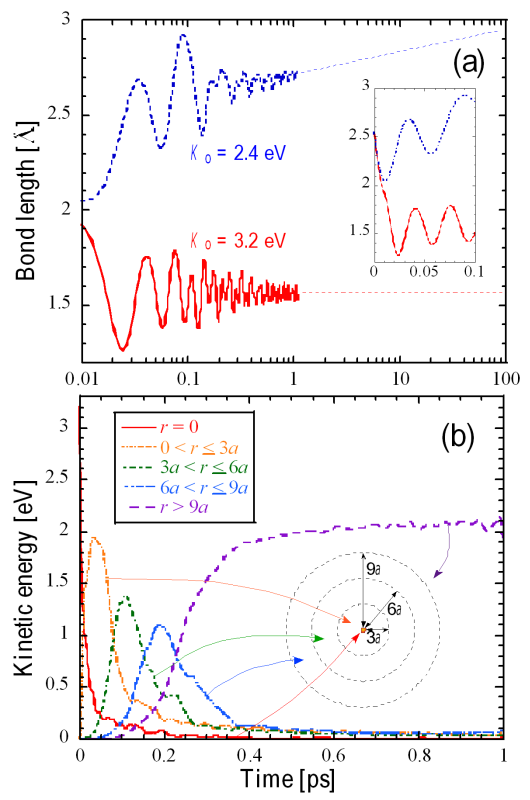

Fig. 8. (a) The bond length between the two opposite carbons as a function of time on a logarithmic scale. The inset shows the same plot from 0 to $0.1 \mathrm{ps}$ on a linear scale. A solid line denotes the case of the initial kinetic energy of $3.2 \mathrm{eV}$ and the initial intrusion of $0.4 \AA$ and a dashed line is the case of the initial kinetic energy of $2.4 \mathrm{eV}$ and the same intrusion. (b) The kinetic energies as a function of time. The solid line indicates the sum of the kinetic energies of two central carbons, and the dotted line indicates that of the carbons located within a $3 a$ radius of the self-localized center except the two central carbons, where $a$ is the lattice constant of the graphite $(1.42 \AA)$. The one-dotted-dashed and twodotted-dashed lines are those of carbons located in the radii between $3 a$ and $6 a$ and between $6 a$ and $9 a$, respectively. The dashed line is that of carbons outside of $9 a$ radius. The corresponding figure about these radii is shown in the inset [14].

Figure $8 \mathrm{a}$ shows the time development of the bond length between the two opposite carbons as a function of time. In the case of $K_{0}=3.2 \mathrm{eV}$, the bond length decreases rapidly from $2.55 \AA$, and settles down gradually at about $1.56 \AA$, repeating vibration. In contrast, the case of $K_{0}=2.4 \mathrm{eV}$ does not result in the bond formation since the system does not overtake the energy barrier to form a bond due to the lack of energy, and returns to the starting graphite without the bond formation.

Figure $8 \mathrm{~b}$ shows the spread of the kinetic energy, $K_{0}=3.2 \mathrm{eV}$, as a function of time. As seen from this figure, the kinetic energy of the two central carbons decreases rapidly in the same time scale as the decrease in the bond length in the inset of Fig. 8a, being spent on overtaking the barrier to form a bond. Simultaneously, the extra energy spreads to the near carbons through the inter-carbon interaction, making the bond stable. Similarly, it moves to more outside carbons one after another. Thus, the kinetic energy which is initially given only to the two central carbons spreads all over the system as time passes. This means that a great number of carbons around the self-localized point act as an energy reservoir to stabilize the bond formation.

We performed the same calculations for various values of the initial energy since it has some width because of the uncertainty for the excitation energy. As a result, the energy of more than about $4.5 \mathrm{eV}$ can overtake the barrier to form the bond. This corresponds to the energy of about three visible photons with $1.57 \mathrm{eV}$. From this energy limitation, the interlayer bond is not always formed even if the self-localization occurs. The electron-hole pair excited in the energy region of $3.3 \pm 1.8 \mathrm{eV}$ self-localizes at the probability of about $2 \%$. Only the component with the energy of more than $4.5 \mathrm{eV}$ among these self-localized states has the possibility to form the interlayer bond, and its ratio is estimated to be about $26 \%$ [18]. In this case, therefore, it is expected that $0.52 \%$ of the excitation contributes to the bond formation.

So far, we have seen the dynamics for the formation of an interlayer bond. However, the diaphite domain includes a large number of inter-layer bonds. Thus, in the next stage, we see how inter-layer bonds are cooperatively formed by further pulse excitations, that is, the effect of multisite excitation [18]. In this stage, we consider the many and random multisite excitations. We choose excited carbon pairs randomly and determine the initial energy of each site randomly, according to a Gaussian distribution with the mean energy, $3.3 \mathrm{eV}$, and the width, $1.8 \mathrm{eV}$. This randomness of the energy originates from the uncertainty of the transient excited state. Due to the AB stacking, there are two types of excited carbon pairs. The first type represents the case where the positions of two carbons at the first layer and at the second one are mutually the same ( $\alpha$ pair). The second type represents the positions shifts in the $x$ direction (the $\beta$ pair). In the latter case, the initial intrusion and velocity of the two excited carbons have not only the $z$ component but also the $x$ component. Assuming only the $x$ polarized light irradiation, all the $\beta$ pairs have the shift only in the $x$ direction.

In this calculation, we perform a two-pulse excitation which means that the system is excited again at a certain time after the first excitation. The pulse excitation induces coherent shearing motion between the graphite layers [19] as seen from Fig. 9a which shows the average shear displacement in $x$ direction as a function of time in the case of the excitation density $6 \%$. As discussed in previous works $[6,7,14,20,21]$, the existence of the shear is important for the diaphite domain formation, because the shear makes the interlayer bonds at $\beta$ pairs ( $\beta$ bonds) stable, which are necessary to build up the 
diaphite structure. Therefore, the $\beta$ bonds are expected to be easily formed when the shear occurs. The two-pulse excitation enables us to confirm it.
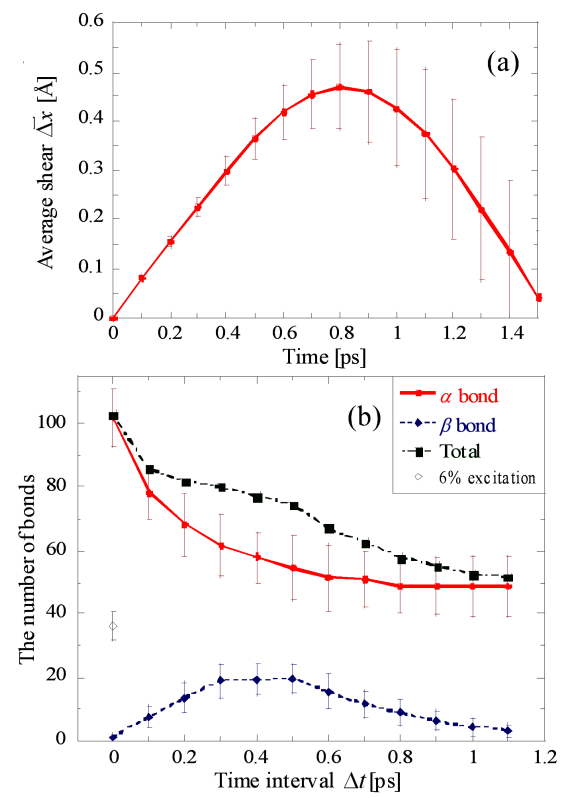

Fig. 9. (a) The average shear displacement in $x$ direction as a function of time. The $6 \%$ excitation is given to the system at $t=0$. It is averaged over all carbon sites and over 50 calculations. (b) The number of interlayer bonds counted at $1.0 \mathrm{ps}$ after the second excitation, as a function of the time interval $\Delta t$ between the first excitation and the second one. The values at $\Delta t=0$ mean no additive excitation, corresponding to the $12 \%$ excitation only at once. The black rhombus at $\Delta t=0$ corresponds to the $6 \%$ excitation only at once [14].

We show the results obtained by the MD calculation for the two-pulse excitation in Fig. 9b. This figure shows the $\Delta t$ dependence on the number of interlayer bonds, where the red and blue solid lines indicate the number of $\alpha$ and $\beta$ bonds, respectively, and the green dashed one is the sum of them. The values at $\Delta t=0$ mean no additive excitation, corresponding to the $12 \%$ excitation only at once. For reference, the black rhombus at $\Delta t=0$ indicates the number of $\alpha$ bonds for the $6 \%$ excitation at once. As seen from this figure, the number of $\alpha$ bonds monotonically decreases and settles down to a constant at more than $\Delta t=0.9 \mathrm{ps}$, while $\beta$ bonds increase until $\Delta t=0.5 \mathrm{ps}$ and afterward decreases gradually. In this calculation, the $\beta$ bonds are formed most efficiently around $\Delta t=0.3-0.5 \mathrm{ps}$. In this time region, the average shear is increasing as seen from Fig. 9a. Therefore, the excitation while the average shear increases is better for the $\beta$ bond formation than the excitation at the maximum of the average shear at $0.8 \mathrm{ps}$. Although the number of $\beta$ bonds remains lower than that of $\alpha$ bonds even in this region, it is essential that $\beta$ bonds can be efficiently formed due to the coherent shear.

We performed the same calculations for various val- ues of the excitation density. Consequently, we found that the number of formed interlayer bonds increases nonlinearly with respect to the number of excited sites due to the cooperative phenomena between excited sites. Through further excitation, new interlayer bonds will be formed more easily around the existing interlayer bonds. Therefore, as the number of these two types of interlayer bonds increases enough, the diaphite will be achieved.

\section{The graphite structure deformation in the vicinity of the diaphite domain}

In order to clarify the effect of the diaphite domain on the surrounding graphite one has to include long-range interactions. Henceforth, we utilize a LCBOP [22] potential of the following form:

$$
E_{b}=\frac{1}{2} \sum_{i j}^{N} V_{i j}^{\mathrm{tot}}=\frac{1}{2} \sum_{i j}^{N}\left(f_{c, i j} V_{i j}^{\mathrm{SR}}+S_{i j} V_{i j}^{\mathrm{LR}}\right),
$$

where $f_{c, i j} V_{i j}^{\mathrm{SR}}$ is the short range interactions potential and $S_{i j} V_{i j}^{\mathrm{LR}}$ is the long range interactions potential.

The short range interactions are of the Brenner type

$$
V_{i j}^{\mathrm{SR}}=V_{\mathrm{R}}\left(r_{i j}\right)-B_{i j} V_{\mathrm{A}}\left(r_{i j}\right),
$$

where

$$
V_{\mathrm{R}}\left(r_{i j}\right)=A \exp (-\alpha r)
$$

and

$$
V_{\mathrm{A}}\left(r_{i j}\right)=B_{1} \exp \left(-\beta_{1} r\right)+B_{2} \exp \left(-\beta_{2} r\right) .
$$

The long range interactions part is of a double Morse potential type:

$$
V^{\mathrm{LR}}(r)=\theta\left(r_{0}-r\right) V_{1}^{\mathrm{M}}(r)+\theta\left(r-r_{0}\right) V_{2}^{\mathrm{M}}(r) .
$$

The $f_{c, i j}$ are smooth cutoff functions

$$
f_{c}(x)=\Theta(-x)+\Theta(x) \Theta(1-x) \exp \left(\frac{\gamma x^{3}}{x^{3}-1}\right),
$$

followed by $S_{i j}$ switching functions excluding first neighbours and defined as:

$$
S_{i j}=1-f_{c, i j} \text {. }
$$

The long range potential is truncated over $6 \AA$ range giving significant numerical advantage over typical two body potentials. The detailed description of the LCBOP potential can be found in [22].

We have investigated how the interlayer distance is changed with respect to the diaphite domain radius. Calculations were performed in the $100 \times 100 \times 12$ carbon atoms slab and the diaphite domain radius was gradually changed from 0 up to 45 . As expected, minimizing the energy with respect to the interlayer distance, we have obtained a significant change of the interlayer distance up to $0.15 \AA$ (Fig. 10).

This rapid change of inter-layer distance may suggest that the appearance of the macroscopic diaphite domain may affect the surrounding graphite. The instability created will result in the shrinkage of graphite inter-layer 


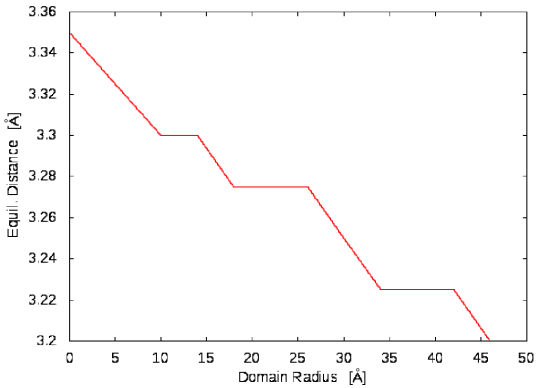

Fig. 10. Interlayer equilibrium distance $[\AA]$ with respect to the diaphite $(L L)$ domain radius $[\AA]$. Calculations were performed using LCBOP [22] potential on the $100 \times 100 \times 12$ graphite slab with the two-layer diaphite domain.

distance resulting in the increase of the overall material density. This effect is desirable in the process of the graphite-diamond conversion.

\section{6. "Bridged-diamond"}

The diaphite although resembling the diamond structure in many aspects is still far from the diamond itself. An interesting question would be if is there another mid-step structure on the way of the graphite-diamond conversion. This structure would have been energetically close enough so it would be accessible by a photoexcitation. We propose that the desired structure is presented in Fig. 11 so called a "bridged-diamond". The structure consists of $s p^{3}$ fully hybridized bonds connected with a "bridge-bond". As a first using LCBOP potential [22] we have optimized the "bridge-bond" as to be $1.48 \AA$ long.

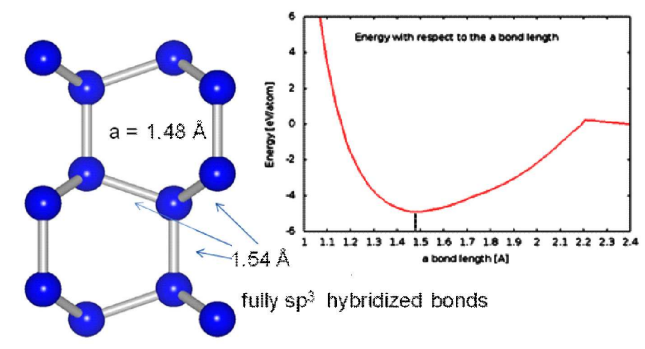

Fig. 11. The "bridged-diamond" structure optimized with LCBOP potential.

Furthermore, using energy a minimization technique and LCBOP potential [22], we have calculated an energy barrier for homogeneous, a two-step graphite-diaphite"bridged-diamond" conversion. We choose that the diaphite structure is represented by the $L 3$ type structure since its bonds geometry is the closest to the $s p^{3}$ hybridization. For a uniform graphite-diaphite conversion (Fig. 12) we have estimated that the energy barrier is $0.15 \mathrm{eV} /$ atom. We have then performed similar calculations for a diaphite-"bridged-diamond" conver- sion resulting in $0.07 \mathrm{eV} /$ atom energy barrier. The combined energy barrier for the two-step graphite-"bridged-diamond" conversion is $0.22 \mathrm{eV} /$ atom. The results suggest that it may be possible for a multistep photoexcited graphite-diaphite-"bridged-diamond" conversion. The authors however do not attempt to explain the mechanism of such transformation only to give qualitative description of possible midstep structures towards diamond configuration.

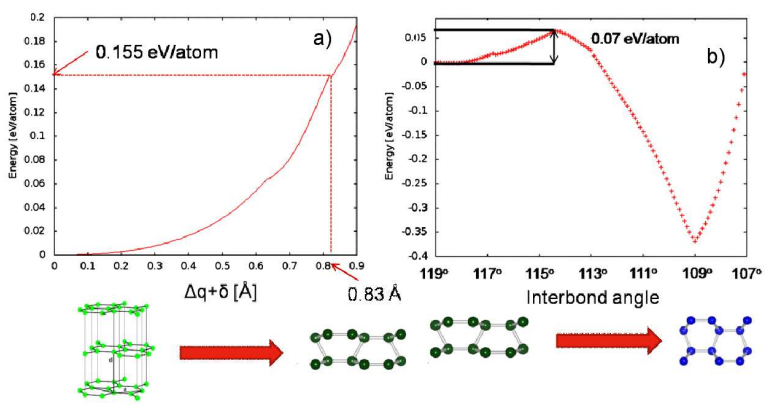

Fig. 12. The adiabatic energy surface of (a) the graphite-diaphite $(L L)$ conversion, (b) the diaphite $(L L)$-"bridged-diamond" conversion.

\section{Final remarks}

The formation of a photoexcited, non-equilibrium, two-layer, a nanoscopic dimerized domain with $\sigma$ inter-layer bonds ("diaphite") in graphite has been investigated. Adiabatic paths between the graphite phase and various (meta) stable domains have been identified and analyzed. These large scale calculations have been performed by means of modified Brenner's and LCBOP empirical potentials. Several possibilities of tanh-like dimerizations have been shown to correspond to the inter-domain energy barrier of an order of $1 \mathrm{eV}$. It has been argued that though the buckling transformation is rather cost full, the planar tanh-like intrusion drastically lowers the transformation energy cost. Also, it has been emphasized that with the help of the photoexcitation it may be possible to conduct the "step-by-step phase conversion". We have also discussed in detail key features of early stages dynamics. We have shown that there exists a finite, non-zero probability that instead of a free carrier relaxation, the photoexcited electron and the hole would remain at the same site. That opens the way to the possible lattice contraction: due to the unbalanced Coulomb interaction between the electron and the hole possible, aforementioned dimerization may occur. The LDA electronic structure analysis reveals that the electronic state of the new phase is an insulator immersed in a semimetal.

Our considerations, complying on one hand with recent experimental observations, open new and underline former questions and problems. The most significant of them are the following two. First, if the photo-stimulated graphite-diamond transformation is going to be considered as a realistic possible scenario, two-layer diaphite 
has to be "foliated" into spatial diamond-like, structure. One of the related problems is the question what is the role of ignored, here, long range interactions. Initial calculations using LCBOP potential suggest increase in the overall material density. Furthermore, there exists another mid step material ("bridged-diamond") towards diamond. This mid step structure is separated from diaphite by rather small energy barrier $(0.07 \mathrm{eV} /$ atom $)$ easily accessible by a photoexcitation. The question about experimental realization of such transformation still remains open.

\section{Acknowledgments}

This work was supported by Grant No. 2290/B/H03/2009/37 by Polish Committee of Science $2009-2012$.

\section{References}

[1] S. Fahy, S.G. Louie, M.L. Cohen, Phys. Rev. B 35 7623 (1987).

[2] S. Fahy, S.G. Louie, M.L. Cohen, Phys. Rev. B 34, 1191 (1986).

[3] F.P. Bundy, J. Chem. Phys. 38, 631 (1963).

[4] T. Irifune, A. Kurio, S. Sakamoto, T. Inoue, H. Sumiya, Nature 421, 599 (2003).

[5] H. Nakayama, H. Yoshida, J. Phys., Condens. Matter 15, R1077 (2003).

[6] L. Radosinski, K. Nasu, J. Kanazaki, K. Tanimura, A. Radosz, T. Luty, in: Molecular Electronic and Related Materials - Control and Probe with Light, Ed. N. Toshio, 2009, Trans-world Research Network Publisher, Kerala, India 2009, Ch. 14.

[7] J. Kanasaki, E. Inami, K. Tanimura, H. Ohnishi, K. Nasu, Phys. Rev. Lett. 102, 087402 (2009).
[8] R.K. Raman, Y. Murooka, C.-Y. Ruan, T. Yang, S. Berber, D. Tomanek, Phys. Rev. Lett. 101, 077401 (2008).

[9] K. Nasu, Photoinduced Phase Transitions, World Sci., Singapore 2004.

[10] A. Radosz, K. Ostasiewicz, P. Magnuszewski, L. Radosinski, F.V. Kusmartsev, J.H. Samson, Phys. Status Solidi B 242, 454 (2005).

[11] A. Radosz, K. Ostasiewicz, P. Magnuszewski, L. Radosinski, F.V. Kusmartsev, J.H. Samson, Phys. Status Solidi B 242, 303 (2005).

[12] A. Radosz, K. Ostasiewicz, P. Magnuszewski, L. Radosinski, F.V. Kusmartsev, J.H. Samson, A.C. Mitus, G. Pawlik, Phys. Rev. E 73, 026127 (2006).

[13] D.W. Brenner, Phys. Rev. B 42, 9458 (1990).

[14] K. Nishioka, K. Nasu, Phys. Rev. B 80, 235420 (2009).

[15] H. Ohnishi, K. Nasu, Phys. Rev. B 79, 054111 (2009).

[16] W.A. Harrison, Electronic Structure and the Properties of Solids: The Physics of the Chemical Bond, Dover Publications, New York 1980.

[17] M. Haibo, C. Fei, L. Chungen, J. Yuansheng, J. Chem. Phys. 122, 104909 (2005).

[18] K. Nishioka, K. Nasu, Phys. Rev. B 82, 035440 (2010).

[19] T. Mishina, K. Nitta, Y. Masumoto, Phys. Rev. B 62, 2908 (2000).

[20] H. Ohnishi, K. Nasu, Phys. Rev. B 80, 014112 (2009).

[21] L. Radosinski, K. Nasu, T. Luty, A. Radosz, Phys. Rev. B 81, 035417 (2010).

[22] J.H. Los, A. Fasolino, Phys. Rev. B 68, 024107 (2003). 